\title{
3-D MRI and DT-MRI Content-adaptive Finite Element Head Model Generation for Bioelectomagnetic Imaging
}

\author{
Tae-Seong Kim and Won Hee Lee \\ Kyung Hee University, Department of Biomedical Engineering \\ Republic of Korea
}

\section{Introduction}

One of the challenges of the $21^{\text {st }}$ century is to understand the functions and mechanisms of the human brain. Although the complexity of deciphering how the brain works is so overwhelming, the electromagnetic phenomenon happening in the brain is one aspect we can study and investigate. In general, this phenomenon of electromagnetism is described as the electrical current produced by action potentials from neurons which are reflected as the changes in electrical potential and magnetic fields (Baillet et al., 2001). These electromagnetic fields of the brain are generally measured with electroencephalogrm (EEG) and magnetoencephalogram (MEG) that are actively used for bioelectromagnetic imaging of the human brain (a.k.a., inverse solutions of EEG and MEG).

In order to investigate the electromagnetic phenomenon of the brain, the human head is generally modelled as an electrically conducting medium and various numerical approaches are utilized such as boundary element method (He et al., 1987; Hamalainen \& Sarvas, 1989; Meijs et al., 1989), finite difference method (Neilson et al., 2005; Hallez et al., 2008), and finite element method (Buchner et al., 1997; Marin et al., 1998; Kim et al., 2002; Lee et al., 2006; Wolters et al., 2006; Zhang et al., 2006; Wendel et al., 2008), to solve the bioelectromagnetic problems (a.k.a., forward solutions of EEG and MEG). Among these approaches, the finite element method (FEM) or analysis (FEA) is known as the most powerful and realistic method with increasing popularity due to (i) readily available computed tomography (CT) or magnetic resonance (MR) images where geometrical shape information can be derived, (ii) recent developments in imaging physical properties of biological tissue such as electrical (Kim et al., 2009) or thermal conductivity, which can be incorporated in to the FE models, (iii) numerical and analytical power that allow truly volumetric analysis, and (iv) much improved computing and graphic power of modern computers.

In applying FEA to the bioelectromagnetic problems, one critical and challenging requirement is the representation of the biological domain (in this case, the human head) as discrete meshes. Although there are some general packages available through which the mesh representation of simple objects is possible, their capability of generating adequate mesh models of biological organs, especially the human head, requires substantial efforts since (i) most mesh generators have some limitations of handling arbitrary geometry of 
complex biological shapes, requiring simplification of complex boundaries, (ii) most mesh generation schemes use a mesh refinement technique to represent fine structures with much smaller elements. This tends to increase number of nodes and elements beyond the computational limit, thus demanding overwhelming computation time, (iii) most mesh generation techniques require careful supervision of users, and (iv) there is a lack of automatic mesh generation techniques for generating FE mesh models for individual heads. Therefore, there is a strong need for fully automatic mesh generation techniques.

In this chapter, we present two novel techniques that automatically generate FE meshes adaptive to the anatomical contents of MR images (we name it as $c M e s h$ ) and adaptive to the contents of anisotropy measured through diffusion tensor magnetic resonance imaging (DTMRI) (we name it as wMesh). The cMeshing technique generates the meshes according to the structural contents of MR images, offering advantages in automaticity and reduction of computational loads with one limitation: its coarse mesh representation of white matter (WM) regions, making it less suitable for the incorporation of the WM tissue anisotropy. The wMeshing technique overcomes this limitation by generating the meshes in the WM region according to the WM anisotropy derived from DT-MRIs. By combining these two techniques, one can generate high-resolution FE head models and optimally incorporate the anisotropic electrical conductivities within the FE head models.

This chapter introduces the cMesh and wMesh methodologies and their evaluations in their effectiveness by comparing the mesh characteristics including geometry, morphology, anisotropy adaptiveness, and the quality of anisotropic tensor mapping into the meshes to those of the conventional FE head models. The presented methodologies offer an automatic high-resolution FE head model generation scheme that is suitable for realistic, individual, and anisotropy-incorporated high-resolution bioelectromagnetic imaging.

\section{Previous Approaches in Finite Element Head Modelling}

Although the classical modelling of the head as a single or multiple spheres (thus called spherical head models) dates back much further than realistic boundary element and finite element head models, the early finite element head modelling was attempted by Yan et al. (1991). Then the later attempts are well summarized in a review paper by Voo et al. (1996). Medical image-based realistic finite element head modelling was introduced a year later by Awada et al. (1997) in 2-D and by Kim et al. (2002) in 3-D. Other than these works, numerous literatures have shown their own approaches of finite element head modelling. Lately, anisotropic properties of brain tissues including white matter and skull have been incorporated into the FE head models and their effects on the forward and inverse solutions have been investigated (Kim et al., 2003; Wolters et al., 2006). Recent studies focus on adaptive mesh modelling, high-resolution mesh generation, and influence of tissue anisotropies. More details can be found in (Lee et al., 2006, 2008; Wolters et al., 2006, 2007).

\section{MRI Content-adaptive Finite Element Head Model Generation}

The procedures of the content-adaptive finite element mesh (cMesh) generation are summarized as follows: namely, (i) MRI content-preserving anisotropic diffusion filtering for noise reduction and feature enhancement, (ii) structural and geometrical feature map generation from the filtered image, (iii) node sampling based on the spatial density of the 
feature maps via a digital halftoning technique, and (iv) mesh generation. The cMesh generation depends on the performance of two key techniques: the quality of feature maps and the accuracy of content-adaptive node sampling. In this study, we focus on the former and its application to MR imagery to build more accurate and efficient cMesh head models for bioelectromagnetic imaging.

\subsection{Gradient Vector Flow (GVF) Nonlinear Anisotropic Diffusion}

To generate an effective and efficient cMesh head model, it is important to remove unnecessary properties of given images such as artifacts and noises. The content-preserving anisotropic diffusion offers pre-segmentation of sub-volumes to simplify the structures of the image and improvement of feature maps where mesh nodes are automatically sampled. In this study, the 3-D Gradient Vector Flow (GVF) anisotropic diffusion algorithm was used (Kim et al., 2003; Kim et al., 2004). The GVF nonlinear diffusion technique, which was successfully applied to regularize diffusion tensor MR images in a previous study (Kim et al., 2004), was proven to be much more robust in comparison to the conventional Structure tensor-based anisotropic diffusion algorithm (Weickert, 1997) and can be summarized as follows.

The GVF as a 3-D vector field can be defined as:

$$
\mathbf{V}(i, j, k)=(\mathbf{u}(i, j, k), \mathbf{v}(i, j, k), \mathbf{w}(i, j, k)) .
$$

The field can be obtained by minimizing the energy functional:

$$
\begin{aligned}
& \varepsilon=\iiint \gamma\left(\eta_{\mathbf{u}}+\eta_{\mathbf{v}}+\eta_{\mathbf{w}}\right)+|\nabla f|^{2}|\mathbf{V}-\nabla f|^{2} \partial x \partial y \partial z \\
& \eta_{\mathbf{u}}=\mathbf{u}_{x}^{2}+\mathbf{u}_{y}^{2}+\mathbf{u}_{z}^{2} \\
& \eta_{\mathbf{v}}=\mathbf{v}_{x}^{2}+\mathbf{v}_{y}^{2}+\mathbf{v}_{z}^{2} \\
& \eta_{\mathbf{w}}=\mathbf{w}_{x}^{2}+\mathbf{w}_{y}^{2}+\mathbf{w}_{z}^{2}
\end{aligned}
$$

where $f$ is an image edge map and $\gamma$ is a noise control parameter.

For 3-D anisotropic smoothing, the Structure tensor $\mathbf{S}$ is formed with the components of $\mathbf{V}$

$$
\mathbf{S}=\mathbf{V}(\mathbf{V})^{T}
$$

The 3-D anisotropic regularization is governed using the GVF diffusion tensor $\mathbf{D}_{\mathrm{GVF}}$ which is computed with eigen components of $\mathbf{S}$.

$$
\partial J / \partial t=\operatorname{div}\left[\mathbf{D}_{G V F} \nabla J\right]
$$

where $J$ is an image volume in 3-D. The regularization behavior of Eq. (4) is controlled with the eigenvalue analysis of the GVF Structure tensor (Ardizzone \& Rirrone, 2003, Kim et al., 2003). 


\subsection{MRI Feature Map Generations}

To generate better feature maps from the filtered images, tensor-driven feature extractors using Hessian tensor (Carmona \& Zhong, 1998; Yang et al., 2003), Structure tensor (AbdElmoniem et al., 2002), and principal curvature methods such as Mean and Gaussian curvature (Gray, 1997; Yezzi, 1998) are utilized. The conventional feature maps proposed by Yang et al. (2003) showed the adequate procedures for the purpose of image representation that meshes are adaptive to the contents of an image where the extraction of image feature information from given image was performed using the Hessian tensor approach.

In the work of Yang et al. (2003), two approaches to generate the feature maps were proposed from the Hessian tensor of each pixel, $\mathbf{H}$ :

$$
\mathbf{H}=\left[\begin{array}{ll}
I(i, j)_{x x} & I(i, j)_{x y} \\
I(i, j)_{y x} & I(i, j)_{y y}
\end{array}\right], I_{x y}=I_{y x}
$$

where $I$ is an image, $i$ and $j$ are image indices, $x$ and $y$ indicate partial derivates in space. One feature map was derived from the maximum of the Hessian tensor components:

$$
f_{\max }(i, j)=\max \left\{\left|I_{x x}(i, j)\right|,\left|I_{x y}(i, j)\right|,\left|I_{y y}(i, j)\right|\right\} .
$$

Another proposed feature map was derived from the eigenvalues, $\mu$ 's, of the tensor:

$$
f_{H \max }(i, j)=\max \left\{\left|\mu_{1}(i, j)\right|,\left|\mu_{2}(i, j)\right|\right\}
$$

The two eigenvalues of the Hessian tensor matrix, denoted by $\mu_{1}$ and $\mu_{2}$ are given by

$$
\begin{aligned}
& \mu_{1}=\frac{1}{2}\left[\left(I_{x x}+I_{y y}\right)+\sqrt{\left(I_{x x}-I_{y y}\right)^{2}+4 I_{x y}^{2}}\right], \\
& \mu_{2}=\frac{1}{2}\left[\left(I_{x x}+I_{y y}\right)-\sqrt{\left(I_{x x}-I_{y y}\right)^{2}+4 I_{x y}^{2}}\right] .
\end{aligned}
$$

The Hessian tensor approach extracts image feature information from the given MR image using the second-order directional derivatives, and its critical attribute is high sensitivity toward feature orientations. However it is known to be highly sensitive toward noise as well.

Currently, advanced differential geometry measures provide better options and choices in deriving feature maps with more effective and accurate properties. In this study, we derived advanced feature maps based on the Hessian and Structure tensor as alternative ways (Lee et al., 2006).

The Hessian tensor-driven feature maps are derived using the eigenvalues of the Hessian tensor in the following way:

$$
f_{\mathbf{H}+}(i, j)=\sqrt{\left(\mu_{1}^{\mathbf{H}}(i, j)+\mu_{2}^{\mathbf{H}}(i, j)\right)},
$$




$$
\begin{gathered}
f_{\mathbf{H}}(i, j)=\sqrt{\mu_{1}^{\mathbf{H}}(i, j)}, \\
f_{\mathbf{H}-}(i, j)=\sqrt{\left(\mu_{1}^{\mathbf{H}}(i, j)-\mu_{2}^{\mathbf{H}}(i, j)\right)},
\end{gathered}
$$

where $\mu$ 's are the positive eigenvalues of the tensor matrix.

Another approach is the use of the the Structure tensor due to robustness in detecting fundamental feature of objects. The Structure tensor $\mathbf{S}$ can be expressed as follows:

$$
\mathbf{S}=\left[\begin{array}{cc}
I_{x}^{2} & I_{x} I_{y} \\
I_{y} I_{x} & I_{y}^{2}
\end{array}\right]
$$

We next derive the Structure tensor-driven feature maps with the eigenvalues of the Structure tensor as the same ways of the Hessian tensor:

$$
\begin{gathered}
f_{\mathbf{S}_{+}}(i, j)=\sqrt{\left(\mu_{1}^{\mathbf{S}}(i, j)+\mu_{2}^{\mathbf{S}}(i, j)\right)}, \\
f_{\mathbf{S}}(i, j)=\sqrt{\mu_{1}^{\mathbf{S}}(i, j)}, \\
f_{\mathbf{S}_{-}}(i, j)=\sqrt{\left(\mu_{1}^{\mathbf{S}}(i, j)-\mu_{2}^{\mathbf{S}}(i, j)\right)} .
\end{gathered}
$$

The above feature map reflects the edges and corners of image structures for the plus sign. By taking the maximum eigenvalue, new feature map can be derived which is a natural extension of the scalar gradient viewed as the value of maximum variations. The other feature map represents the local coherence or anisotropy for the minus sign (Tschumperle \& Deriche, 2002).

In addition, we generate new feature maps via the principal curvature. There are geometric meanings with respect to the eigenvalues and eigenvectors of the tensor matrix. The first eigenvector (corresponding eigenvalue represents the largest absolute value) is the direction of the greatest curvature. Conversely, the second eigenvector is the direction of least curvature. Also its eigenvalue has the smallest absolute value. The consistent eigenvalues are the respective amounts of these curvatures. The eigenvalues of tensor matrix with real values indicate principal curvatures, and are invariant under rotation.

The Mean curvature can be obtained from the Hessian tensor matrix (Gray, 1997; Yezzi, 1998). It is equal to the half of the trace of $\mathbf{H}$ which is invariant to the selection of $x$ and $y$ as well. The new feature map $f_{M}$ using the Mean curvature can be expressed as follows:

$$
f_{M}(i, j)=\frac{I_{x x}\left(1+I_{y}^{2}\right)-2 I_{x} I_{y} I_{x y}+I_{x y}\left(1+I_{x}^{2}\right)}{2\left(1+I_{x}^{2}+I_{y}^{2}\right)^{3 / 2}} .
$$


From the Hessian tensor again, we also derive another feature map $f_{G}$ using the Gaussian curvature as shown below:

$$
f_{G}(i, j)=\frac{I_{x x} I_{y y}-I_{x y}^{2}}{\left(1+I_{x}^{2}+I_{y}^{2}\right)^{2}} .
$$

\subsection{Node Sampling via Digital Halftoning}

In order to produce content-adaptive mesh nodes based on the spatial information of the feature map, we utilize the following popular digital halftoning algorithm. The FloydSteinberg error diffusion technique with the serpentine scanning is applied to create content-adaptive nodes in accordance with the spatial density of image feature maps (Floyd \& Steinberg, 1975). This algorithm produces more nodes in the high frequency regions of the image. The sensitivity of feature map is controlled by regenerating a new feature map with the parameter, $\kappa$ as shown below. In this way, the total number of content-adaptive nodes generated by the halftoning algorithm can be adjusted.

$$
f^{\prime}(i, j)=f(i, j)^{1 / \kappa}
$$

where $f$ is a feature map and $\kappa$ is a control parameter for the number of content-adaptive nodes.

\subsection{FE Mesh Generation}

Once cMesh nodes are generated from the procedures described above, FE mesh generation using triangular elements in 2-D and tetrahedral elements in 3-D is performed using the Delaunay tessellation algorithm (Watson, 1981).

\subsection{Isotropic Electrical Conductivity in cMesh}

In order to assign electrical properties to the tissues of the head, we segment the MR images into five sub-regions including white matter, gray matter, CSF, skull, and scalp. BrainSuite2 (Shattuck \& Leahy, 2002) is used for the segmentation of the different tissues within the head. The first step is to extract the brain tissues from MR images other than the skull, scalp, and undesirable structures. Then, the brain images are classified into each tissue region including white mater, gray matter, and CSF using a maximum a posterior classifier (Shattuck \& Leahy, 2002). The skull and scalp compartments are segmented using the skull and scalp extraction technique based on a combination of thresholding and morphological operations such as erosion and dilation (Dogdas et al., 2005).

The following isotropic electrical conductivity values according to each tissue type are used: white matter $=0.14 \mathrm{~S} / \mathrm{m}$, gray matter $=0.33 \mathrm{~S} / \mathrm{m}, \mathrm{CSF}=1.79 \mathrm{~S} / \mathrm{m}$, scalp $=0.35 \mathrm{~S} / \mathrm{m}$, and skull $=0.0132 \mathrm{~S} / \mathrm{m}$ respectively (Kim et al., 2002; Wolters et al., 2006). 


\subsection{Analysis on the MRI Content-adaptive Meshes}

\subsubsection{Numerical Evaluation of cMeshes: Feature Maps and Mesh Quality}

In order to investigate the effects of the feature maps on cMeshes, we used the following five indices as the goodness measures of content-adaptiveness: (i) correlation coefficient (CC) of the feature map to the original MRI, (ii) root mean squared error (RMSE), (iii) relative error (RE) between the original MRI and the reconstructed MRI based on the nodal MR intensity values (Lee et al., 2006), (iv) number of nodes, and (v) number of elements. For fair comparison of the content-adaptiveness of cMeshes, almost same number of meshes were generated by adjusting the mesh parameter $\kappa$ as in Eq. (19). To test the content information of the non-uniformly placed nodes, the MR images were reconstructed using the MR spatial intensity values at the sampled nodes via the cubic interpolation method. Then the RMSE and RE values were calculated between the original and reconstructed MR images.

We next performed the numerical evaluations of cMesh quality, since the mesh quality highly affects computational analysis in terms of numerical accuracy on the solution on FEA. The evaluation of mesh quality is critical, since it provides some indications and insights of how appropriate a particular discretization is for the numerical accuracy on FEA. For example, as the shapes of elements become irregular (i.e, the angles of elements are highly distorted), the error of the discretization in the solutions of FEA is increased and as angles in an element become too small, the condition number of the element matrix is increased, thus the numerical solutions of FEA are less accurate. The geometric quality indicators were used for the investigation of cMesh quality as the mesh quality measures (Field, 2000). For a triangle element in 2-D, the mesh quality measure can be expressed as

$$
q=\alpha \frac{A}{l_{1}^{2}+l_{2}^{2}+l_{3}^{2}}
$$

where $A$ represents the area of the triangle, and $l_{1}, l_{2}$, and $l_{3}$ are the edge lengths of the triangle element, and $\alpha=4 \sqrt{3}$ is a normalizing coefficient justifying the quality of an equilateral triangle to 1 (i.e., $q=1$, when $l_{1}=l_{2}=l_{3}$. If $q>0.6$, the triangle possesses acceptable mesh quality). The overall mesh quality was evaluated for triangle elements in terms of the arithmetic mean by

$$
Q_{a}=\frac{1}{N} \sum_{i=1}^{N} q_{i}
$$

where $N$ indicates the number of elements.

Additionally, we counted the elements with the poor quality (i.e., $q<0.6$ ) as an indicator of the poor elements that affect the overall mesh quality. Certainly, other measures are available using other geometric quality indicators (Berzins, 1999).

Fig. 1 shows a set of results from 2-D cMesh generation obtained using the conventional techniques by Yang et al. (2003). Fig. 1(a) is a MR image, (b) conventional feature map obtained using $f_{\max }$ and (c) another suggested feature map using $f_{\text {Hmax }}$. Fig. 1(d) shows content-adaptive nodes from Fig. 1(c). Figs. 1(e) and (f) show content-adaptive meshes in 2D from Figs. 1(b) and (c) respectively. There are 2327 nodes and 4562 triangular elements in 
Fig. 1(e) and 2326 nodes and 4560 elements in Fig. 1(f). The triangle with different sizes indicates adaptive characteristics of mesh generation in accordance with the two different feature maps.

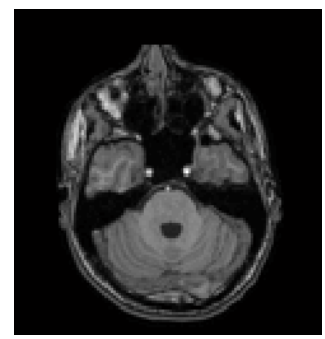

(a)

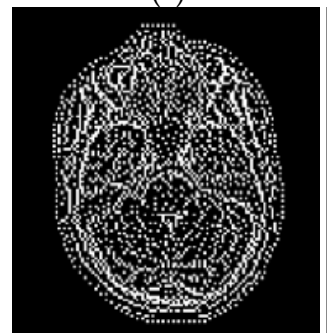

(d)

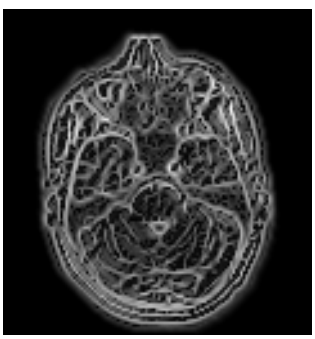

(b)

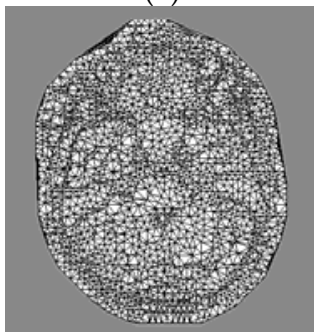

(e)

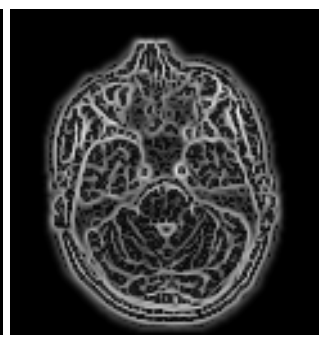

(c)

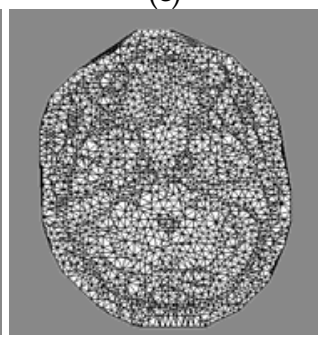

(f)

Fig. 1. Feature maps and cMeshes of a MR image: (a) a MR image, (b) feature map from (a) using $f_{\max },(\mathrm{c})$ using $f_{\mathrm{Hmax}},(\mathrm{d})$ content-adaptive nodes from (c), (e) cMeshes from (b) with 2327 nodes and 4562 elements, and (f) cMeshes from (c) with 2326 nodes and 4560 elements.

We also generated the cMeshes of the given MRI using the advanced feature maps. Figs. 2(a)-(c) display the feature maps obtained using $f_{\mathrm{H}^{+}}, f_{\mathrm{H}}$, and $f_{\mathrm{H}-}$ derived from the Hessian approach. Their corresponding cMeshes are shown in Figs. 2 (d)-(f) respectively. There are 2326 nodes and 4560 elements in Fig. 2(d), 2324 nodes and 4556 elements in Fig. 2(e), and 2329 nodes and 4566 elements in Fig. 2(f). The high sensitivity of Hessian tensor to the structures of MRI is clearly visualized.

Fig. 3 shows a set of demonstrative results from the Structure tensor approaches. Figs. 3 (a)(c) show the improved feature maps acquired using $f_{\mathrm{S}^{+}}, f_{\mathrm{S}}$, and $f_{\mathrm{S}^{-}}$respectively. The corresponding cMeshs are shown in Figs. 3 (d)-(f). There are 2323 nodes and 4554 elements in Fig. 3(d), 2325 nodes and 4558 elements in Fig. 3(e), and 2323 nodes and 4554 elements in Fig. 3(f) respectively. Based on these results, it indicates that the Structure tensor-driven feature extractor yields optimal information on image features and their resultant cMeshes look most adaptive to the contents of the given MRI. That is larger elements are present in the homogeneous regions and smaller elements in the high frequency regions with reasonable numbers of nodes and elements. Content-adaptive nature is clearly visible in the contents of the given cMeshes. 


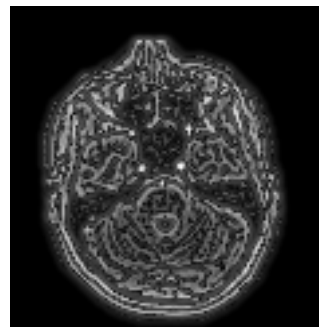

(a)

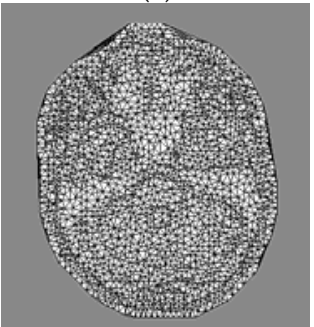

(d)

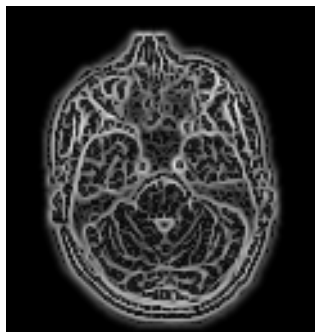

(b)

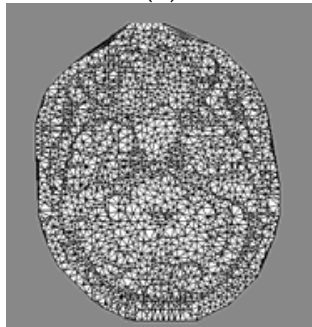

(e)

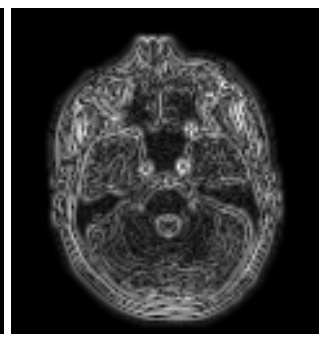

(c)

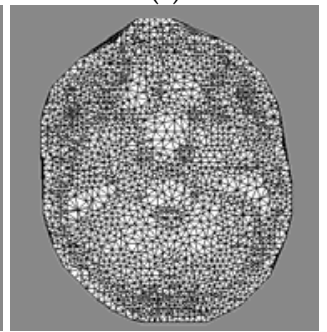

(f)

Fig. 2. Hessian tensor-derived feature maps and cMeshes: (a) feature map using $f_{\mathbf{H}^{+}}(\mathrm{b})$ using $f_{\mathrm{H}}$ (c) using $f_{\mathrm{H}-,}(\mathrm{d})$ cMeshes from (a) with 2326 nodes and 4560 elements, (e) cMeshes from (b) with 2324 nodes and 4556 elements, (f) cMeshes from (c) with 2329 nodes and 4566 elements.

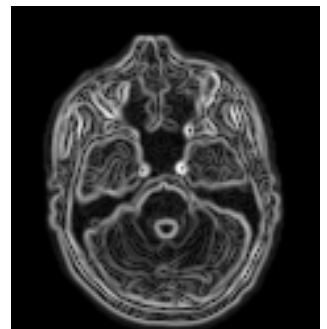

(a)

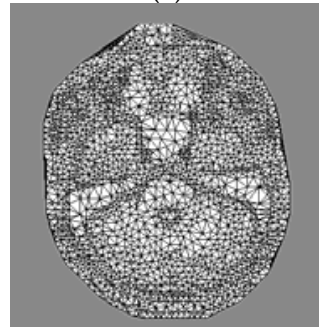

(d)

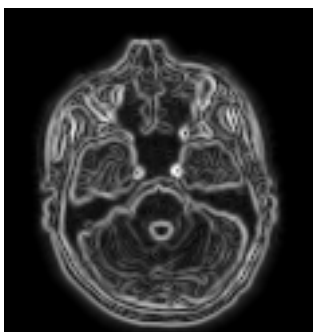

(b)

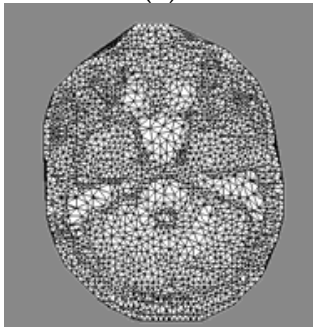

(e)

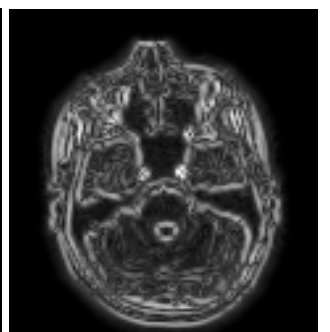

(c)

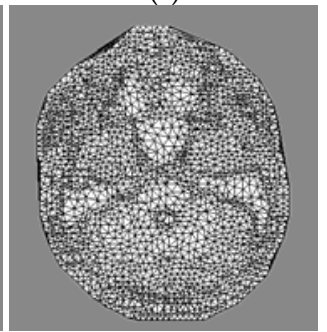

(f)

Fig. 3. Structure tensor-derived feature maps and cMeshes: (a) feature map using $f_{\mathrm{S}+}$ (b) using $f_{\mathrm{S}}$, (c) using $f_{\mathrm{s}-,}$ (d) cMeshes from (a) with 2323 nodes and 4554 elements, (e) cMeshes from (b) with 2325 nodes and 4558 elements, (f) cMeshes from (c) with 2323 nodes and 4554 elements. 
In addition, by using the Mean and Gaussian curvature, the feature maps obtained using $f_{\mathrm{M}}$ and $f_{\mathrm{G}}$ are shown in Figs. 4(a) and (b) respectively. The resultant cMeshes are shown in Figs. $4(c)$ and (d). The characteristics of curvatures to the image features are clearly noticeable too.

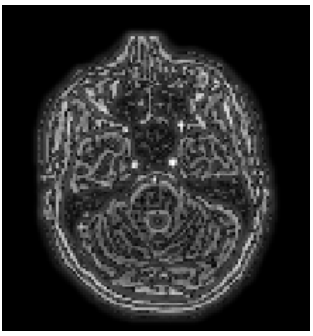

(a)

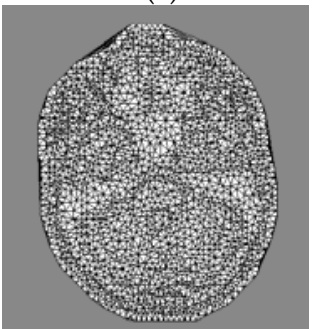

(c)

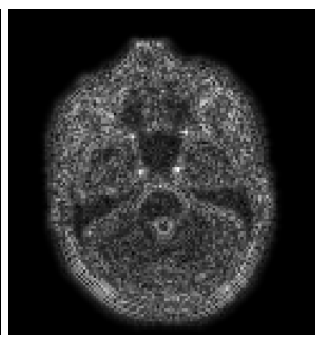

(b)

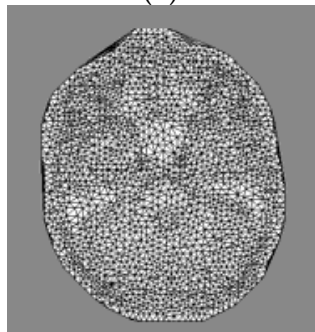

(d)

Fig. 4. Curvature-derived feature maps and cMeshes: (a) feature map using $f_{\mathrm{M}}$, (b) using $f_{\mathrm{G}}$, (c) cMeshes from (a) with 2326 nodes and 4560 elements, (d) cMeshes from (b) with 2325 nodes and 4558 elements.

The CC values in Table 1 show strong correlation between the Structure tensor-driven feature map and the original MRI, indicating the Structure-driven feature extractor generates much better content-adaptive features. Although the CC value of Structure tensordriven approach is lower than the feature maps by $f_{\mathrm{H}+}, f_{\mathrm{H}-}, f_{\mathrm{M}}$, and $f_{\mathrm{G}}$, it produced much lower RMSE and RE values, indicating the reconstructed MRI is much closer to the original MRI. As for the cMesh quality, the result by $f_{\mathrm{G}}$ describes the highest value. Also, the Structure tensor approach show greatly acceptable values with much lower number of poor elements compared to other feature extractors, indicating the Structure tensor-driven approach will offer numerically accurate and efficient computational accuracy in FEA.

\subsubsection{Numerical Evaluation of cMeshes: Regular Mesh vs. cMesh}

To evaluate numerical accuracy of the cMesh head model on FEA in 3-D against the conventional regular FE model commonly used in E/MEG forward or inverse problems, two 3-D cMesh models of the whole head (matrix size: $128 \times 128 \times 77$, spatial resolution: $1 \times 1 \times 1$ $\mathrm{mm}^{3}$ ) differing in their mesh resolution were built using the Structure tensor-based (i.e., $f_{\mathrm{s}+}$ ) cMesh generation technique as described earlier. For the reference model, the regular mesh head model was generated as the gold standard using fine and equidistant tetrahedral elements with inner-node spacing of $2 \mathrm{~mm}$, since analytical solutions cannot be obtained for an arbitrary geometry of the real head. 
The numerical quality of the cMesh head models were evaluated by comparing the scalp forward potentials computed from the cMesh models against those of the regular mesh model. To solve EEG forward problems governed by the Poisson's equation under the quasistatic approximation of the Maxwell's equation (Sarvas, 1987), the FE head models along with isotropic electrical conductivity information were imported into a software ANSYS (ANSYS, Inc., PA, USA). The forward potential solutions due to the identical current generator (Yan et al., 1991; Schimpf et al., 2002) were obtained using the preconditioned conjugate gradient solver of ANSYS. Then the scalp potential values from the cMesh head models were compared to those from the reference FE head model. As evaluation measures, both CC and RE were used along with the forward computation time (CT) as a numerical efficiency measure.

Fig. 5 shows a set of results from the 3-D regular and cMesh models of the whole head with isotropic electrical conductivities. In Figs. 5(a)-(c), there are 159,513 nodes and 945,881 tetrahedral elements in the regular FE head model. The cMesh model of the entire head with 109,628 nodes and 694,588 tetrahedral elements is given in Figs. 5(d)-(f). The mesh generation time for the 3-D regular and cMesh head models was $169.5 \mathrm{sec}$ and $68.1 \mathrm{sec}$ respectively on a PC with Pentium-IV CPU 3.0 GHz and 2GB RAM. In comparison to the regular mesh model in Figs. 5(a)-(c), the content-adaptive meshes are clearly visible according to MR structural information in Figs. 5(d)-(f). Various mesh sizes indicate the adaptive characteristics of meshes based on given MR anatomical contents as shown in Figs. $5(\mathrm{~d})-(\mathrm{f})$.

\begin{tabular}{|c|c|c|c|c|c|c|c|}
\hline \multirow[t]{2}{*}{ Method } & \multirow[t]{2}{*}{$\begin{array}{l}\text { No. of } \\
\text { Nodes }\end{array}$} & \multirow[t]{2}{*}{$\begin{array}{c}\text { No. of } \\
\text { Elements }\end{array}$} & \multirow{2}{*}{$\begin{array}{c}\text { MRI vs. } \\
\text { Feature } \\
\text { Map }\end{array}$} & \multicolumn{2}{|c|}{$\begin{array}{c}\text { MRI vs. } \\
\text { Reconstructed MRI }\end{array}$} & \multirow[t]{2}{*}{$\begin{array}{l}\text { cMesh } \\
\text { Quality }\end{array}$} & \multirow{2}{*}{$\begin{array}{l}\text { No. of Poor } \\
\text { Elements: } \\
\quad q<0.6\end{array}$} \\
\hline & & & & RMSE & RE & & \\
\hline$f_{\max }$ & 2327 & 4562 & 0.45 & 40.11 & 0.21 & $\begin{array}{ll}0.79 & \pm \\
0.16 & \end{array}$ & 503 \\
\hline$f_{\text {Hmax }}$ & 2326 & 4560 & 0.49 & 46.98 & 0.25 & $\begin{array}{ll}0.78 & \pm \\
0.16 & \end{array}$ & 617 \\
\hline$f_{\mathrm{H}^{+}}$ & 2326 & 4560 & 0.68 & 34.94 & 0.18 & $\begin{array}{ll}0.80 & \pm \\
0.15 & \end{array}$ & 418 \\
\hline$f_{\mathbf{H}}$ & 2324 & 4556 & 0.50 & 46.38 & 0.24 & $\begin{array}{ll}0.78 & \pm \\
0.16 & \end{array}$ & 649 \\
\hline$f_{\mathrm{H}-}$ & 2329 & 4566 & 0.62 & 34.94 & 0.18 & $\begin{array}{ll}0.82 & \pm \\
0.14 & \end{array}$ & 224 \\
\hline$f_{\mathrm{S}^{+}}$ & 2323 & 4554 & 0.60 & 31.96 & 0.17 & $\begin{array}{ll}0.81 & \pm \\
0.14 & \end{array}$ & 284 \\
\hline$f_{\mathrm{S}}$ & 2325 & 4558 & 0.61 & 31.93 & 0.17 & $\begin{array}{ll}0.82 & \pm \\
0.14 & \end{array}$ & 243 \\
\hline$f_{\text {s- }}$ & 2323 & 4554 & 0.61 & 32.96 & 0.17 & $\begin{array}{ll}0.82 & \pm \\
0.14 & \end{array}$ & 254 \\
\hline$f_{\mathrm{M}}$ & 2326 & 4560 & 0.68 & 34.94 & 0.18 & $\begin{array}{ll}0.80 & \pm \\
0.15 & \end{array}$ & 418 \\
\hline$f_{\mathrm{G}}$ & 2325 & 4558 & 0.70 & 28.96 & 0.14 & $\begin{array}{ll}0.83 & \pm \\
0.13 & \end{array}$ & 166 \\
\hline
\end{tabular}

Table 1. Numerical evaluations of the content-adaptiveness of cMeshes. 


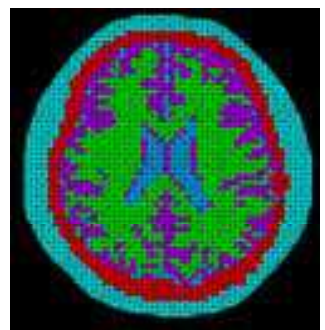

(a)

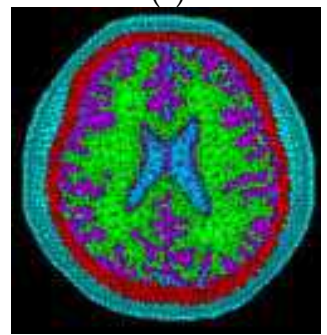

(d)

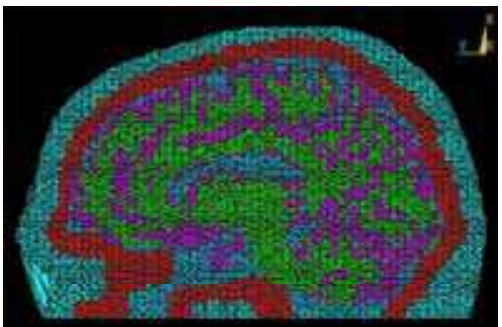

(b)

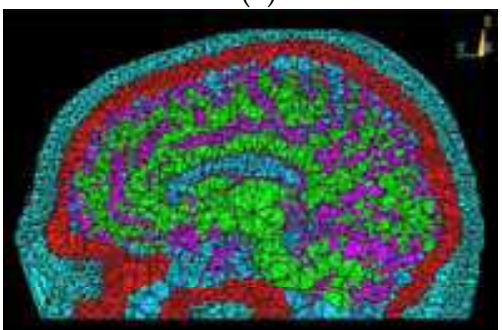

(e)

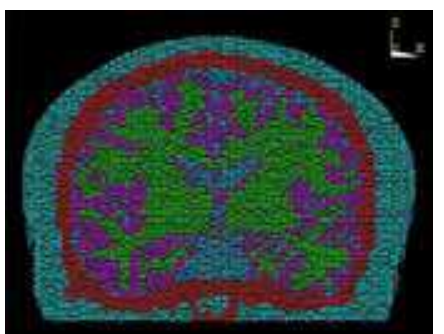

(c)

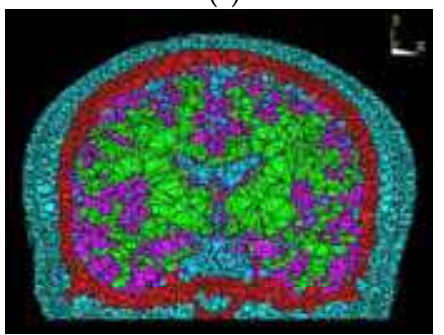

(f)

Fig. 5. Comparison of geometrical mesh morphology of the 3-D FE models of the whole head. Top row shows (a) a transaxial slice, (b) sagittal cutplane, and (c) coronal view from the regular mesh head model with 159,513 nodes and 945,881 tetrahedral elements through the five sub-regions segmented. Bottom row displays (d) a tranaxial slice, (e) sagittal cutplane, and (f) coronal view from the cMesh head model with 109,628 nodes and 694,588 elements. (cyan: scalp, red: skull, green: white matter, purple: gray matter, and deepskyblue: CSF).

Figs. 6(a) and (b) display the sagittal cutplanes of the 3-D forward potential maps from the regular FE (i.e., reference) and cMesh head model of the whole head respectively. The minor differences of the EEG electrical potential distribution between the regular vs. cMesh head models are directly noticeable in Figs. 6(a) and (b). In Table 2, the CC values show strong correlation of the scalp electrical potentials between the cMesh head models and reference model. The results from cMesh- 2 show $\mathrm{CC}=0.999$ and $\mathrm{RE}=0.037$, indicating there is only minor difference in the scalp electrical potentials but significant gain in CT of 55\% (5.47 to $3.02 \mathrm{~min}$ ) with significantly reduced nodes and elements.

\begin{tabular}{|c|c|c|c|c|c|}
\hline FE model & No. of Nodes & No. of Elements & CC & RE & CT (min) \\
\hline Reference & 159,513 & 945,881 & 1 & 0 & $1(5.47)$ \\
\hline cMesh-1 & 148,852 & 943,072 & 0.999 & 0.031 & $0.60(3.28)$ \\
\hline cMesh-2 & 109,628 & 694,588 & 0.999 & 0.037 & $0.55(3.02)$ \\
\hline
\end{tabular}

Table 2. Numerical quality of the scalp electrical potentials in the cMesh head models. 


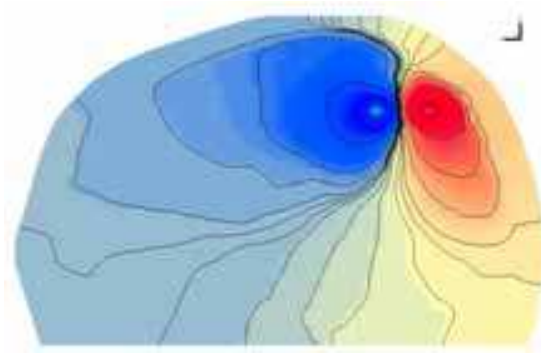

(a)

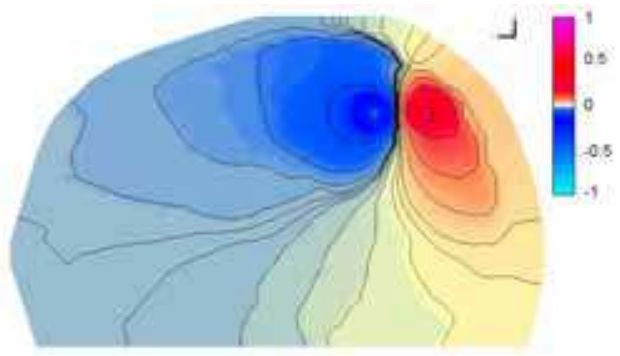

(b)

Fig. 6. Sagittal view of the 3-D forward potential maps from (a) the reference FE head model and (b) the cMesh-1 head model. The resultant EEG forward potentials are normalized by the maximum value of the EEG potential for isopotential visualization.

\section{DT-MRI Content-adaptive Finite Element Head Model Generation}

Fig. 7 describes the schematic steps of building wMesh head models along with the generation of the cMesh head model. The detailed technical steps are explained in the subsequent sections.

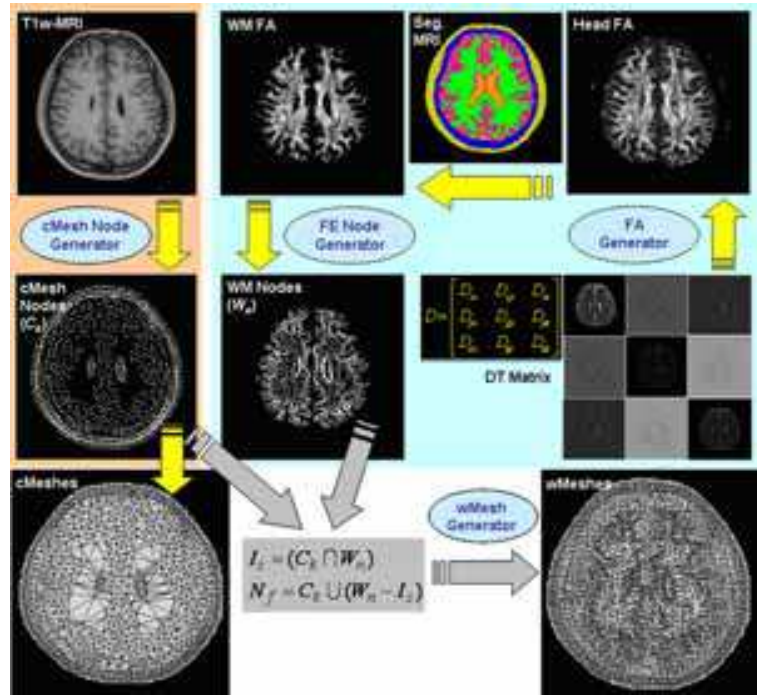

Fig. 7. Schematic diagram of generating a cMesh and wMesh head model.

\subsection{DT-MRI Feature Map Generation}

From DT-MRI data, the symmetric DT matrix is obtained: namely, the diffusion components along the $\mathrm{x}-\mathrm{y}$ direction, the $\mathrm{x}-\mathrm{z}$ direction, and the $\mathrm{y}-\mathrm{z}$ direction (i.e., $D_{x y}, D_{x z}$, and $D_{y z}$ ) in addition to the traditional measurements of diffusivities along the $\mathrm{x}-, \mathrm{y}-$, and $\mathrm{z}$-axes (i.e., $D_{x x}$, $D_{y y}$, and $D_{z z}$ ) (Bihan et al., 2001). The mathematical representation of the DT matrix is shown in Fig. 7. 
For the wMesh head modeling, fractional anisotropy (FA) as an anisotropy feature map is used. The FA map is calculated using the eigenvalues of the DT matrix as follows:

$$
F A=\frac{\sqrt{3}}{\sqrt{2}} \frac{\sqrt{\left(\lambda_{1}-\lambda\right)^{2}+\left(\lambda_{2}-\lambda\right)^{2}+\left(\lambda_{3}-\lambda\right)^{2}}}{\sqrt{\lambda_{1}^{2}+\lambda_{2}^{2}+\lambda_{3}^{2}}}, 0 \leq F A \leq 1
$$

where $\lambda_{1}, \lambda_{2}$, and $\lambda_{3}$ are three eigenvalues and $\lambda$ is the average of the eigenvalues.

The FA measures the ratio of the anisotropic part of the DT over the total magnitude of the tensor (Bihan et al., 2001). The minimum value of FA can occur only in a perfectly isotropic medium. The maximum value arises only when $\lambda_{1}>\lambda_{2}=\lambda_{3}$. The FA is widely used to represent the anisotropy of the DT due to its robustness against to noise.

\section{2 wMesh Generation}

To build the wMesh head model, the first step is to co-register a set of $\mathrm{T}_{1}$-weigthed MRIs to DT-MRIs using a voxel similarity-based affine registration technique (Maes et al., 1997). Then to generate the WM anisotropy-adaptive nodes, the head FA maps are derived from the measured DT matrix using Eq. (22). The WM FA maps are extracted from the head FA maps using the information of the WM regions segmented from the structural MRIs. To create the WM anisotropy-adaptive nodes based on the WM FA maps where the strong anisotropy is present, the node sampling is performed according to the spatial anisotropic density of the FA maps via the Floyd-Steinberg error diffusion algorithm technique (Floyd \& Steinberg, 1975). Basically more nodes are created in the high anisotropic density regions of the FA maps.

In addition to the node generation in the WM regions based on the anisotropy feature maps, the cMesh nodes are generated from the $\mathrm{T}_{1}$-weighted MRIs using our $\mathrm{cMesh}$ node generator as described in the previous sections. For the generation of the wMesh head models (see Fig. 7), the cMesh nodes $C_{k}$ and $W M$ nodes $W_{n}$ are used which are expressed as:

$$
\begin{aligned}
& C_{k}(x, y, z)=\{k \mid 1 \leq k \leq N\}, \\
& W_{n}(x, y, z)=\{n \mid 1 \leq n \leq M\},
\end{aligned}
$$

where $k$ and $n$ are the nodal indices, $x, y$, and $z$ the nodal coordinates in the Euclidean space, and $N$ and $M$ the total number of nodes of cMesh nodes $C_{k}$ and $W M$ nodes $W_{n}$ respectively. We find the intersectional node information (i.e., identical nodal positions, $I_{s}$ ) of $C_{k}$ and $W_{n}$, using Eq. (25), since they share the same position of FE nodes which are overlapped in both the cMesh and WM node maps.

$$
\begin{gathered}
I_{s}(x, y, z)=\left(C_{k} \cap W_{n}\right), \\
I_{s}(x, y, z)=\left\{s \mid s \in\left(C_{k} \cap W_{n}\right)\right\},
\end{gathered}
$$

where $s$ denotes the nodal indices intersected. 
Then we compute the wMesh nodes $N_{f}$ in the following way:

$$
N_{f}(x, y, z)=C_{k}(x, y, z) \bigcup\left[W_{n}(x, y, z)-I_{s}(x, y, z)\right] .
$$

The computed wMesh nodes $N_{f}$ (i.e., the superfluous FE nodes $I$ were removed) are used to generate the wMesh head model. The dense nodes in the WM regions are produced according to the WM anisotropic density over the cMesh nodes $C_{k}$. Once the wMesh nodes $N_{f}$ are sampled from the procedures described above, the FE mesh generation using tetrahedral elements in 3-D is done via the Delaunay tessellation algorithm (Watson, 1981) to construct the wMesh head models. Fig. 7 shows the distinct mesh characteristics in the WM regions between the cMesh and wMesh head models.

\subsection{Anisotropic Electrical Conductivity in wMesh}

To set up the anisotropic electrical conductivity tensors in the WM tissue, we first hypothesize that the electrical conductivity tensors share the eigenvectors with the measured diffusion tensors according to the work of Basser et al. (2004). Then, we have adopted two different techniques of modeling WM anisotropy conductivity derived from the measured diffusion tensors: (i) a fixed anisotropic ratio in each WM voxel (Wolters et al., 2006) and (ii) a variable anisotropic ratio using a linear conductivity-to-diffusivity relationship in combination with a constraint on the magnitude of the electrical conductivity tensor (Hallez et al., 2008). Two different approaches of deriving the WM anisotropic conductivity tensors are briefly described as below.

To derive the WM anisotropic conductivity tensor with a fixed anisotropic ratio, the anisotropic conductivity tensor $\sigma$ of the WM compartments is expressed as:

$$
\sigma=\mathbf{S} \operatorname{diag}\left(\sigma_{\text {long }}, \sigma_{\text {trans }}, \sigma_{\text {trans }}\right) \mathbf{S}^{-1}
$$

where $\mathbf{S}$ is the orthogonal matrix of unit length eigenvectors of the measured DT at the barycenter of the WM FEs. $\sigma_{\text {long }}$ and $\sigma_{\text {trans }}$ denote the eigenvalues parallel (longitudinal) and perpendicular (transverse) to the fiber directions, respectively, with $\sigma_{\text {long }} \geq \sigma_{\text {trans }}$.

Then we computed the longitudinal and transverse eigenvalues (i.e., anisotropic ratio of $\sigma_{\text {long }}$ and $\sigma_{\text {trans }}$ ) using the volume constraint (Wolters et al., 2006) retaining the geometric mean of the eigenvalues. The volume of the conductivity tensor is calculated as follows:

$$
\frac{4}{3} \pi \sigma_{\text {iso }}^{3}=\frac{4}{3} \pi \sigma_{\text {long }} \sigma_{\text {trans }}^{2}
$$

The anisotropic FE head models differing in the anisotropic ratio (i.e., 1:2, 1:5, 1:10, and 1:100) are generated using different conductivity tensor eigenvalues under the volume constraint algorithm, Eq. (29).

To compute the WM anisotropic conductivity tensors with the variable (or proportional) anisotropic ratios, a linear scaling approach of the diffusion tensor ellipsoids is used according to the self-consistent effective medium approach (EMA) (Sen et al., 1989; Tuch et 
al., 1999; 2001). EMA states a linear relationship between the eigenvalues of the conductivity tensor $\sigma$ and the eigenvalue of diffusion tensor $d$ in the following way:

$$
\sigma=\frac{\sigma_{e}}{d_{e}} d
$$

where $\sigma_{e}$ and $d_{e}$ represent the extracellular conductivity and diffusivity respectively (Tuch et al., 2001). This approximated linear relationship assumes the intracellular conductivity to be negligible (Tuch et al., 2001; Haueisen et al., 2002). According to the proposition by Hallez et al. (2008), the scaling factor $\sigma_{e} / d_{e}$ can be computed using the volume constraint in Eq. (29) as shown below.

The linear relationship between the conductivity tensor eigenvalues and diffusion tensor eigenvalues in the $\mathrm{WM}$ regions can be represented as

$$
\frac{d_{1}}{\sigma_{1}}=\frac{d_{2}}{\sigma_{2}}=\frac{d_{3}}{\sigma_{3}}
$$

where $d_{1}, d_{2}$, and $d_{3}$ are the eigenvalues of the diffusion tensor at each WM voxel. $\sigma_{1}, \sigma_{2}$, and $\sigma_{3}$ are the unknown eigenvalues of the electrical conductivity tensor at the corresponding voxel. Then the volume constraint algorithm as in Eq. (29) can be applied to compute the anisotropic electrical conductivities. The volume constraint equation can be rewritten as follows:

$$
\frac{4}{3} \pi \sigma_{i s o}^{3}=\frac{4}{3} \pi \sigma_{1} \sigma_{2} \sigma_{3}
$$

where $\sigma_{1}$ is the eigenvalues to the largest eigenvector. $\sigma_{2}$ and $\sigma_{3}$ represent the eigenvalues to the perpendicular eigenvectors, respectively.

\subsection{Analysis on DT-MRI Content-adaptive Meshes}

\subsubsection{Comparison of Anisotropy Adaptiveness and Anisotropy Tensor Mapping}

To examine the effectiveness of the wMesh head model, we tested both anisotropy adaptiveness and the quality of anisotropic mapping into the meshes by comparing to the regular mesh and cMesh head models.

Fig. 8 shows a set of exemplary results from a regions of interest (ROI) to compare the anisotropy adaptiveness of the FE head models to the given mesh morphology. Fig. 8(a) shows a transaxial $\mathrm{T}_{1}$-weighted MRI. The ROI, enclosing $38 \times 38$ voxels, is highlighted with a box in red on the $\mathrm{T}_{1}$-weighted MRI. The enlarged ROI of the $\mathrm{T}_{1}$-weighted MRI and its corresponding color-coded FA map derived from the DTs are given in Figs. 8(b) and (c) respectively. In Fig. 8(c), the projections of the principal tensor directions on the ROI colorcoded FA map are visualized with white lines. Figs. 8(d)-(f) show the ROI regular meshes, cMeshes, and wMeshes respectively. In contrast to the regular meshes in Fig. 8(d), the anisotropy-adaptive characteristics of the wMeshes according to the WM anisotropy information is clearly noticeable in Fig. 8(f). Moreover, it appears that there is higher mesh 
density in the WM regions where the degree of the anisotropy is strongly present. The results from wMeshes demonstrate that mapping the WM electrical anisotropy into the meshes could be performed more accurately. As mentioned previously, cMeshes in Fig. 8(e) show too coarse mesh characteristics in the WM tissues, which seem be unsuitable for the incorporation of the WM tensor anisotropy.

We next examined the quality of anisotropy mapping into the meshes which could be important since the correct representation of anisotropy affects the accuracy of FEA. Fig. 9 illustrates the projection of the DT ellipsoids overlaid on the transaxial slice of a $\mathrm{T}_{2}$-weighted MRI. Fig. 9(a) displays the original DT ellipsoids in the WM tissues. In the corresponding WM regions, the DT ellipsoids at the barycenters of the WM elements from the wMeshes are shown in Fig. 9(b). The diameters in any directions of the DT ellipsoids reflect the diffusivities in their corresponding directions, and their major principle axes are oriented in the directions of maximum diffusivities. As observed in Fig. 9(d), the wMeshes are likely to provide a better way of reflecting the details of the directionality and magnitude of the anisotropic tensors due to the dense mesh features and anisotropy-adaptive characteristics in the WM regions. In other words, the wMesh head model better incorporate the WM anisotropic electrical conductivities and thereby the errors of the anisotropy modeling could be reduced.

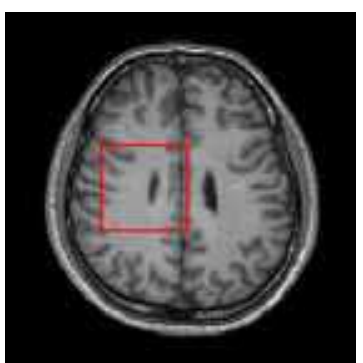

(a)

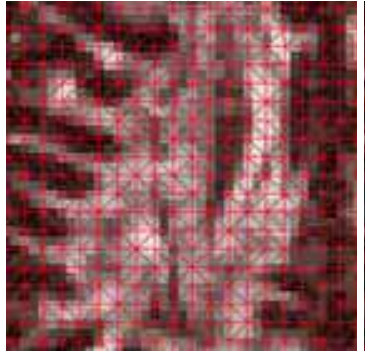

(d)

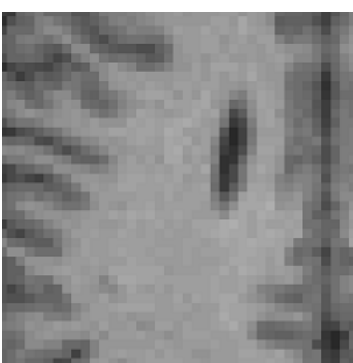

(b)

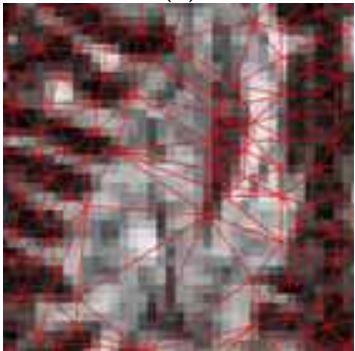

(e)

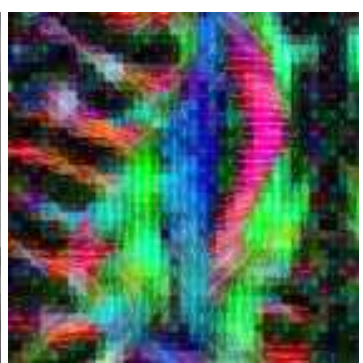

(c)

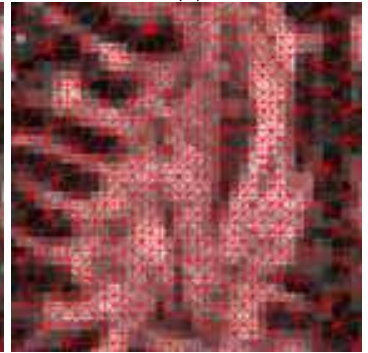

(f)

Fig. 8. Anisotropy adaptiveness of the FE head models. (a) a transaxial $\mathrm{T}_{1}$-weighted MR slice including the red box which indicates the regions of interest (ROI), (b) a ROI (38 x 38 voxels) of the $\mathrm{T}_{1}$-weighted MRI, (c) corresponding color-coded FA with the projections of the principal tensor directions shown as white lines, (d) regular meshes overlaid on the FA map, (e) cMeshes, and (f) wMeshes. 


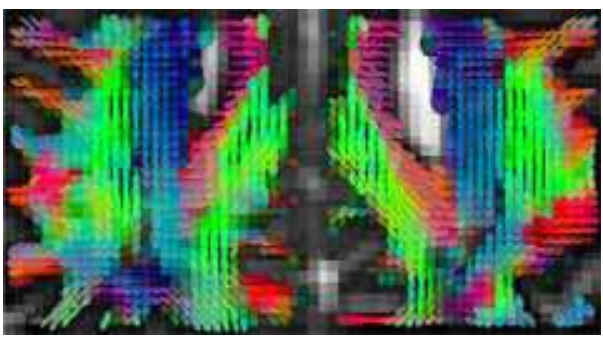

(a)

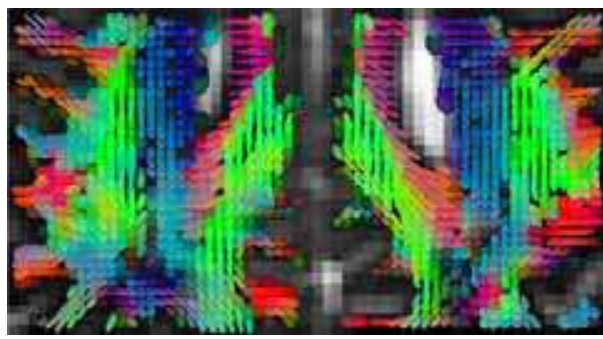

(b)

Fig. 9. Mapping the DT ellipsoids of the WM regions onto a transaxial cut of the $\mathrm{T}_{2}$-weighted MRI: (a) the original DT ellipsoids and (b) DT ellipsoids in the barycenters of the WM elements from the wMesh head model. The color indicates the orientation of the principal tensor eigenvector (red: mediolateral, green: anteroposterior, and blue: superoinferior direction).

\subsubsection{Effect of Anisotropic Electrical Conductivity}

To study the effects of the WM anisotropic electrical conductivity on the EEG forward solutions, we compared the EEG electrical potentials from the anisotropic wMesh head models against those of the isotropic models. To obtain the EEG forward potentials, we solved the Poisson's equation (Sarvas, 1987) due to the following current sources (Yan et al., 1991; Schimpf et al., 2002): as superficial sources, (i) an approximately tangentially oriented source (the posterior-anterior direction) and (ii) a radially oriented source (the inferiorsuperior direction) in the cortex; as a deep source (iii) an approximately radial source in the thalamus. Each dipole was placed in the isotropic gray matter regions with careful attention, since EEG fields are particularly sensitive to the conductivity changes of the brain tissue next to the dipole (Haueisen et al., 1997; Gencer \& Acar, 2004).

Fig. 10 visualizes the wMesh model of the whole head through the five sub-regions segmented. There are 160,230 nodes and 1,009,440 tetrahedral elements in the wMesh head model. The fully automatic generation of the wMeshes took $80.3 \mathrm{sec}$ on a PC with PentiumIV CPU 3.0 GHz and 2GB RAM. Figs 10(a)-(c) display the transaxial, sagittal, and coronal view of the head model respectively. The wMeshes in Fig. 10 show dense and adaptive meshes in the WM regions generated based on the WM FA information. It is also seen that compared to the regular FE head model in Fig. 5(a)-(c), there are much smoother boundaries of the meshes at the skin, outer, and inner regions, thus possibly avoiding the stair-step approximation of curved boundaries (e.g., Wolters et al., 2007) and reducing EEG forward modeling errors. The WM anisotropy-adaptive meshing technique offers an optimal way of incorporating the WM anisotropic conductivity tensors into the meshes. 


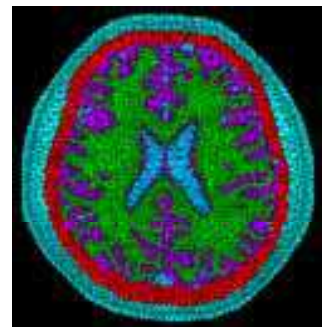

(a)

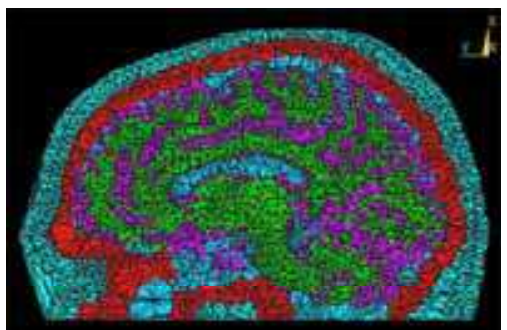

(b)

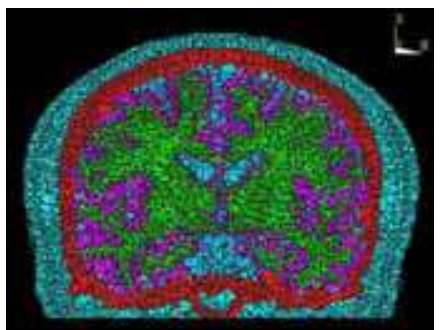

(c)

Fig. 10. Visualization of the 3-D wMesh model of the whole head with 160,230 nodes and 1,009,440 tetrahedral elements (color labeling as described in Fig. 5): (a) a transaxial slice, (b) sagittal cutplane, and (c) coronal view.

Fig. 11 displays the results of the EEG forward potential maps from the wMesh models of the whole head. According to the given source types, the resultant EEG forward distributions of the sagittal and coronal views from the isotropic wMesh models are visualized in Figs. 11(a)-(c) respectively. The EEG potential maps from the anisotropic wMesh head models at the 1:10 fixed anisotropic ratio are shown in Figs. 11(d) and (e). Fig. 11(f) shows the EEG potential distributions from the wMesh model at the anisotropic ratio of 1:100. Based on the observation in Fig. 11, the differences of the EEG electrical potential distributions between the isotropic vs. anisotropic wMesh models are directly noticeable through the altering directions and extension of the isopotential lines. In particular, the isopotentials in Fig. 11(f) show the greater effects of the WM anisotropic conductivities due to the strong anisotropy of 1:100.

To evaluate the numerical differences of the EEG forward solutions between the isotropic vs. anisotropic wMesh models, the scalp potential values were quantitatively compared using two similarity measures: relative difference measure (RDM) and magnification factor (MAG). Meijs et al., (1999) introduced these metrics to quantify the topography and magnitude errors. The quantitative results of the scalp electrical potentials according to different anisotropy settings are given in Table 3.

The results from the wMesh head model with the 1:10 anisotropic ratio using the tangential dipole show that the inclusion of the WM anisotropy resulted in the low RDM value of 0.037 and the MAG value of 0.959 . On the other hand, a slightly larger influence (RDM=0.046 and MAG=0.910) was found in the wMesh model with the 1:10 anisotropic ratio for the radial dipole, thus indicating that the WM anisotropy led to the topography errors of the EEG and weakened the EEG fields. Moreover, the strong effects by the 1:100 WM anisotropy ratio were observed in the MAG value of 0.427 , describing the WM anisotropy strongly weakened the EEG potential fields. The WM anisotropic conductivities around the deep source have a greater influence on the EEG forward solutions. In the case of the anisotropic models by the variable anisotropy setting, the results show smaller differences on the EEG forward solutions due to much lower variable anisotropic ratios of the WM anisotropic electrical conductivities. 


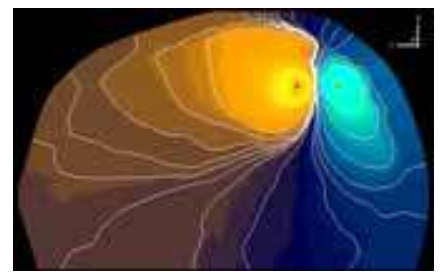

(a)

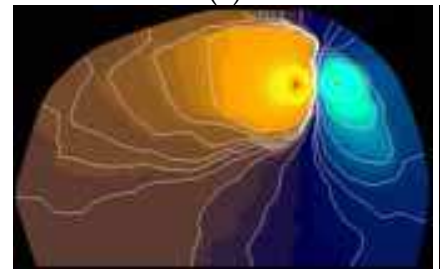

(d)

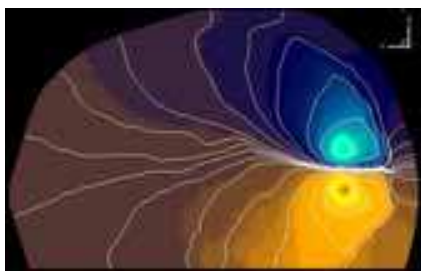

(b)

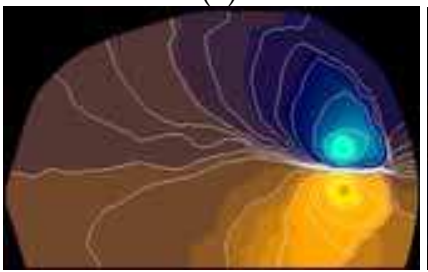

(e)

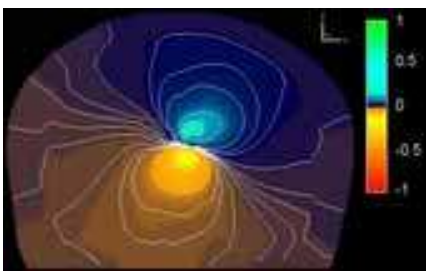

(c)

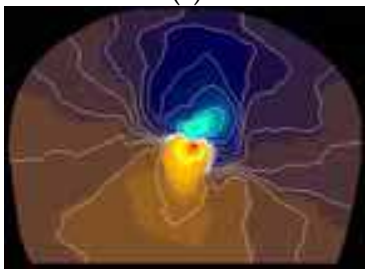

(f)

Fig. 11. EEG forward potential maps of the isotropic vs. anisotropic wMesh models of the whole head: Top row from the isotropic models, (a) the sagittal cutplane with a tangentially oriented dipole, (b) with a radially oriented dipole, and (c) coronal view with a deep source. Bottom row from the anisotropic models, (d) sagittal cutplane with a tangentially oriented dipole with the fixed anisotropic ratio of 1:10, (e) with a radially oriented dipole at the 1:10 fixed anisotropic ratio, and (f) coronal view with a deep source with the 1:100 fixed anisotropic ratio. The resultant EEG forward potentials are normalized by the maximum value of the EEG potential for isopotential visualization.

\begin{tabular}{|c|c|c|c|c|c|c|c|}
\hline \multirow{2}{*}{ Anisotropy } & \multirow{2}{*}{ Ratio } & \multicolumn{2}{|c|}{ Tangential } & \multicolumn{2}{c|}{ Radial } & \multicolumn{2}{c|}{ Deep } \\
\cline { 3 - 8 } & & RDM & MAG & RDM & MAG & RDM & MAG \\
\hline \multirow{3}{*}{ Fixed } & $1: 2$ & 0.010 & 0.998 & 0.012 & 0.992 & 0.012 & 0.988 \\
\cline { 2 - 8 } & $1: 5$ & 0.024 & 0.983 & 0.030 & 0.957 & 0.053 & 0.924 \\
\cline { 2 - 8 } & $1: 10$ & 0.037 & 0.959 & 0.046 & 0.910 & 0.111 & 0.838 \\
\cline { 2 - 8 } & $1: 100$ & 0.080 & 0.790 & 0.153 & 0.640 & 0.540 & 0.427 \\
\hline \multicolumn{2}{|c|}{ Variable } & 0.022 & 0.992 & 0.022 & 0.986 & 0.045 & 0.982 \\
\hline
\end{tabular}

Table. 3. Numerical differences of the scalp electical potentials between the isotropic vs. anisotorpic wMesh head models.

\section{Conclusion}

In this chapter, we have introduced how to generate MRI content-adaptive FE meshes (i.e., cMesh) and DT-MRI anisotropy-adaptive FE meshes (i.e., wMesh) of the human head in 3-D. These cMesh and wMesh generation methodologies are fully automatic with the presegmented boundary information of the sub-regions of the head (such as gray matter, white matter, CSF, skull, and scalp), DT information, and conductivity values of the segmented regions. Although the choice of using cMesh or wMesh depends on the aim of each FEA, the combination of these meshes should allow high-resolution FE modelling of the head. Also the presented technique should be extendable to other parts of the human body and their FEA of bioelectromagnetic phenomenon thereof. 


\section{Acknowledgement}

This work was supported by a grant of Korea Health 21 R\&D Project, Ministry of Health and Welfare, Republic of Korea (02-PJ3-PG6-EV07-0002). This work was also supported by the Korea Science and Engineering Foundation (KOSEF) grant funded by the Korea government (MEST) (2009-0075462).

\section{References}

Abd-Elmoniem, K. Z.; Youssef, A. M. \& Kadah, Y. M. (2002). Real-time speckle reduction and coherence enhancement in ultrasound imaging via nonlinear anisotropic diffusion. IEEE Trans. Biomed. Eng., Vol. 49, No. 9, 997-1014, 0018-9294

Ardizzone, E. \& Rirrone, R. (2003). Automatic segmentation of MR images based on adaptive anisotropic filtering, Proceedings of IEEE Int. Conf. Image Ana. Process. (ICIAP'03), pp. 283-288, 0-7695-1948-2, Italy, Sept., 2003, IEEE

Awada, K. A.; Jackson, D. R.; Baumann, S. B.; Williams, J. T.; Wilton, D. R.; Baumann, S. B. \& Papanicolaou, A. C. (1997). Computational aspects of finite element modeling in EEG source localization. IEEE Trans. Biomed. Eng., Vol. 44, No. 8, 736-752, 0018-9294

Baillet, S.; Mosher, J. C. \& Leahy, R. M. (2001). Electromagnetic brain mapping. IEEE Sig. Process. Mag., Nov., 14-30, 1053-5888

Basser, P. J.; Mattiello, J. \& Bihan, D. L. (1994). MR diffusion tensor spectroscopy and imaging. Biophys. J. Vol. 66, 259-67, 0006-3495

Berzins, M. (1999). Mesh quality: a function of geometry, error estimates or both?. Eng. with Comp., Vol. 15, 236-247, 0177-0667

Bihan, D. L.; Mangin, J. F.; Poupon, C.; Clark, C. A.; Pappata, S.; Molko, N. \& Chabriat, H. (2001). Diffusion tensor imaging: concepts and applications. J. MRI, Vol. 37, 534-546, 1053-1807

Buchner, H.; Knoll, G.; Fuchs, M.; Rienaker, A.; Beckmann, R.; Wagner, M.; Silny, J. \& Pesch, J. (1997). Inverse localization of electric dipole current sources in finite element models of the human head. Electroenceph. Clin. Neurophysiol., Vol. 102, 267-278, 1388-2457

Carmona, R. A. \& Zhong, S. (1998). Adaptive smoothing respecting feature directions. IEEE Trans. Image Process., Vol. 7, No. 3, 353-358, 1057-7149

Dogdas, B.; Shattuck, D. W. \& Leahy, R. M. (2005). Segmentation of skull and scalp in 3-D human MRI using mathematical morphology. Hum. Brain Mapping, Vol. 26, 273-285, 1065-9471

Field, D. A. (2000). Qualitative measures for initial measures. Int. J. Numer. Meth. Eng., Vol. $47,887-906,0029-5981$

Floyd, R. \& Steinberg, L. (1975). An adaptive algorithm for spatial gray scale. in SID Int. Symp. Digest of Tech. 36-37, 0003-966X

Gencer, N. G. \& Acar, C. E. (2004). Sensitivity of EEG and MEG measurements to tissue conductivity. Phys. Med. Biol., Vol. 49, 701-717, 0031-9155

Gray, A. (1997). The gaussian and mean curvatures and surfaces of constant gaussian curvature. \$16.5 and Ch. 21 in Modern Differential Geometry of Curves and Surfaces with Mathematica, 2nd ed. Boca Raton, FL: CRC Press, 373-380 and 481-500, 1997 
Hallez, H.; Vanrumste, B.; Hese, P. V.; Delputte, S. \& Lemahiueu, I. (2008). Dipole estimation errors due to differences in modeling anisotropic conductivities in realistic head models for EEG source analysis. Phys. Med. Biol., Vol. 53, 1877-1894, 0031-9155

Hamalainen, M. S. \& Sarvas, J. (1989). Realistic conductivity geometry model of the human head for interpretation of neuromagnetic data. IEEE Trans. Biomed. Eng., Vol. 36, No. 2, 165-171, 0018-9294

Haueisen, J.; Ramon, C.; Brauer, H. \& Nowak, H. (1997). Influence of tissue resistivities on neuromagnetic fields and electric potentials studied with a finite element model of the head. IEEE Trans. Biomed. Eng., Vol. 44, No. 8, 727-735, 0018-9294

Haueisen, J.; Tuch, D. S.; Ramon, C.; Schimpf, P. H.; Wedeen, V. J.; George, J. S. \& Belliveau, J. W. (2002). The influence of brain tissue anisotropy on human EEG and MEG. NeuroImage, Vol. 15, 159-66, 1053-8119

He, B.; Musha, T.; Okamoto, Y.; Homma, S.; Nakajima, Y. \& Sato, T. (1987). Electric dipole tracing in the brain by means of the boundary element method and its accuracy. IEEE Trans. Biomed. Eng., Vol. 34, No. 6, 406-414, 0018-9294

Katsavounidis, I. \& Kuo, C-C. J. (1997). A multiscale error diffusion technique for digital halftoning. IEEE Trans. Image Process., Vol. 6, No. 3, 483-490, 1057-7149

Kim, S.; Kim, T.-S.; Zhou, Y. \& Singh, M. (2003). Influence of conductivity tensors on the scalp electrical potential: study with 2-D finite element models. IEEE Trans. Nucl. Sci. Vol. 50, No. 1, 133-138, 0018-9499

Kim, T.-S.; Zhou, Y.; Kim, S. \& Singh, M. (2002). EEG distributed source imaging with a realistic finite-element head model. IEEE Trans. Nucl. Sci., Vol. 49, No. 3, 745-752, 0018-9499

Kim, T.-S.; Jeong, J.; Shin, D.; Huang, C.; Singh, M. \& Marmarelis, V. Z. (2003). Sinogram enhancement for ultrasonic transmission tomography using coherence enhancing diffusion, Proceedings of IEEE Int. Symposium on Ultrasonics, pp. 1816-1819, 0-78037922-5, Hawaii, USA, Oct., 2004, IEEE

Kim, T.-S.; Kim, S.; Huang, D. \& Singh, M. (2004). DT-MRI regularization using 3-D nonlinear gradient vector flow anisotropic diffusion, Proceedings of Int. Conf. IEEE Eng. Med. Biol., pp. 1880-1883, 0-7803-8439-3, San Francisco, USA, Sep., 2004, IEEE

Kim, H. J.; Kim, Y. T.; Minhas, A. S.; Jeong, W. C.; Woo, E. J.; Seo, J. K. \& Kwon, O. J. (2009). In vivo high-resolution conductivity imaging of human leg using MREIT: the first human experiment. IEEE Trans. Med. Imag., Vol. 28, No. 1, 0278-0062

Lee, W. H.; Kim, T.-S.; Cho, M. H.; Ahn, Y. B. \& Lee, S. Y. (2006). Methods and evaluations of MRI content-adaptive finite element mesh generation for bioelectromagnetic Problems. Phys. Med. Biol., Vol. 51, No. 23, 6173-6186, 0031-9155

Lee, W. H.; Seo, H. S.; Kim, S. H.; Cho, M. H.; Lee, S. Y. \& Kim, T.-S. (2008). Influnce of white matter anisotropy on the effects of transcranial direct current stimulation: a finite element study, Proceedings of Int. Conf. Biomed. Eng., pp. 460-464, 978-3-540-92840-9, Singapore, Dec., 2008, Springer Berlin Heidelberg.

Maes, F.; Collignon, A.; Vandermeulen, D.; Marchal, G.; Marchal, G. \& Suetens, P. (1997). Multimodality image registration by maximization of mutual information. IEEE Trans. Med. Imag., Vol. 16, 187-198, 0278-0062

Marin, G.; Guerin, C.; Baillet, S.; Garnero, L. \& Meunier, G. (1998). Influence of skull anisotropy for the forward and inverse problem in EEG: simulation studies using FEM on realistic head models. Hum. Brain Mapp., Vol. 6, 250-269, 1065-9471 
Meijs, J. W. H.; Weier, O. W.; Peters, M. J. \& Oosterom, A. V. (1989). On the numerical accuracy of the boundary element method. IEEE Trans. Biomed. Eng., Vol. 36, No. 10, 1038-1049, 0018-9294

Neilson, L. A.; Kovalyov, M. \& Koles, Z. J. (2005). A computationally efficient method for accurately solving the EEG forward problem in a finely discretized head model. Clin. Neurophysiol., Vol. 116, 2302-2314, 1388-2457

Sarvas, J. (1987). Basic mathematical and electromagnetic concepts of the biomagnetic inverse problem. Phys. Med. Biol., Vol. 32, 11-22, 0031-9155

Schimpf, P.; Ramon, C. \& Haueisen, J. (2002). Dipole models for the EEG and MEG. IEEE Trans. Biomed. Eng.. Vol. 49, No. 5, 409-418, 0018-9294

Sen, A. K. \& Torquato, S. (1989). Effective electrical conductivity of two-phase disordered anisotropic composite media. Phys. Rev. B. Condens. Matter., Vol. 39, 4504-4515, 1098-0121

Shattuck, D. W. \& Leahy, R. M. (2002). BrainSuite: an automated cortical surface identification tool. Med. Image Anal., Vol. 8, 129-142, 1361-8415

Tschumperle, D. \& Deriche, R. (2002). Diffusion PDEs on vector-valued images. IEEE Sig. Proc. Mag., Sep., 16-25, 1053-5888

Tuch, D. S.; Wedeen, V. J.; Dale, A. M.; George, J. S. \& Belliveau J. W. (1999). Conductivity mapping of biological tissue using the diffusion MRI. Ann. N. Y. Acad. Sci., Vol. 888, 314-316, 0077-8923

Tuch, D. S.; Wedeen, V.; Dale, A.; George, J. \& Belliveau, J. (2001). Conductivity tensor mapping of the human brain using diffusion tensor MRI. Proc. Natl. Acad. Sci. USA, Vol. 98, 11697-11701, 0027-8424

Voo, V.; Kumaresan, S.; Pintar, F. A.; Yoganandan, N. \& Sances, A. (1996). Finite-element models of the human head. Med. Biol. Eng. Comput., Vol. 34, No. 5, 375-381, 01400118

Watson, D. F. (1981). Computing the n-dimensional Delaunay tessellation with application to Voronoi polytypes. The Comp. Jour., Vol. 24, No. 2, 167-172, 1460-2067

Weickert, J. (1997). A review of nonlinear diffusion filtering, In: Scale-Space Theory in Computer Vision, Romeny, B. ter Haar., Florack L, Koenderink J, Vierver M (Ed.) Vol. 1252, 3-28, Springer Berlin, 978-3-540-63167-5

Wendel, K.; Narra, N. G.; Hannula, M.; Kauppinen, P. \& Malmivuo, J. (2008). The influence of CSF on EEG sensitivity distributions of multilayered head models. IEEE Trans. Biomed. Eng., Vol. 55, No. 4, 1454-1456, 0018-9294

Wolters, C. H.; Anwander, A.; Tricoche, X.; Weinstein, D.; Koch, M. A. \& MacLeod, R. S. (2006). Influence of tissue conductivity anisotropy on EEG/MEG field and return current computation in a realistic head model: a simulation and visualization study using high-resolution finite element modeling. NeuroImage, Vol. 30, 813-826, 10538119

Wolters, C. H.; Anwander, A.; Berti, G. \& Hartmann, U. (2007). Geometry-adapted hexahedral meshes improve accuracy of finite-element-method-based EEG source analysis IEEE Trans. Biomed., Eng. Vol. 54, No. 8, 1446-1153, 0018-9294

Yan, Y.; Nunez, P. L. \& Hart, R. T. (1991). Finite-element model of the human head: scalp potentials due to dipole sources. Med. Biol. Eng. Comput., Vol. 29, 475-481, 0140-0118 
Yang, Y.; Wernick, M. N. \& Brankov, J. G. (2003). A fast approach for accurate contentadaptive mesh generation. IEEE Trans. Image Process., Vol. 12, No. 8, 866-881, 10577149

Yezzi, A. (1998). Modified curvature motion for image smoothing and enhancement. IEEE Trans. Image Process., Vol. 7, No. 3, 345-352, 1057-7149

Zhang, Y. C.; Ding, L.; van Drongelen, W.; Hecox, K.; Frim, D. M. \& He, B. (2006). A cortical potential imaging study from simultaneous extra- and intracranial electrical recordings by means of the finite element method. NeuroImage Vol. 31, 1513-1524, 1053-8119 


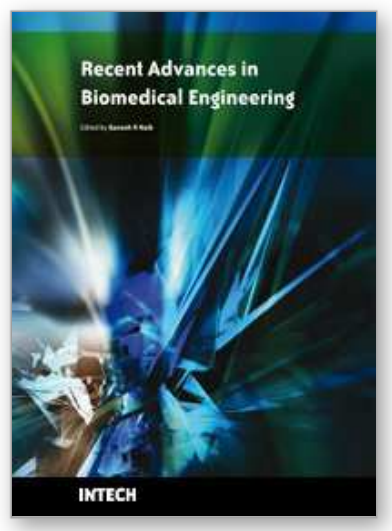

\author{
Recent Advances in Biomedical Engineering \\ Edited by Ganesh R Naik
}

ISBN 978-953-307-004-9

Hard cover, 660 pages

Publisher InTech

Published online 01, October, 2009

Published in print edition October, 2009

The field of biomedical engineering has expanded markedly in the past ten years. This growth is supported by advances in biological science, which have created new opportunities for development of tools for diagnosis and therapy for human disease. The discipline focuses both on development of new biomaterials, analytical methodologies and on the application of concepts drawn from engineering, computing, mathematics, chemical and physical sciences to advance biomedical knowledge while improving the effectiveness and delivery of clinical medicine. Biomedical engineering now encompasses a range of fields of specialization including bioinstrumentation, bioimaging, biomechanics, biomaterials, and biomolecular engineering. Biomedical engineering covers recent advances in the growing field of biomedical technology, instrumentation, and administration. Contributions focus on theoretical and practical problems associated with the development of medical technology; the introduction of new engineering methods into public health; hospitals and patient care; the improvement of diagnosis and therapy; and biomedical information storage and retrieval. The book is directed at engineering students in their final year of undergraduate studies or in their graduate studies. Most undergraduate students majoring in biomedical engineering are faced with a decision, early in their program of study, regarding the field in which they would like to specialize. Each chosen specialty has a specific set of course requirements and is supplemented by wise selection of elective and supporting coursework. Also, many young students of biomedical engineering use independent research projects as a source of inspiration and preparation but have difficulty identifying research areas that are right for them. Therefore, a second goal of this book is to link knowledge of basic science and engineering to fields of specialization and current research. The editor would like to thank the authors, who have committed so much effort to the publication of this work.

\title{
How to reference
}

In order to correctly reference this scholarly work, feel free to copy and paste the following:

Tae-Seong Kim and Won Hee Lee (2009). 3-D MRI and DT-MRI Content-adaptive Finite Element Head Model Generation for Bioelectomagnetic Imaging, Recent Advances in Biomedical Engineering, Ganesh R Naik (Ed.), ISBN: 978-953-307-004-9, InTech, Available from: http://www.intechopen.com/books/recent-advances-inbiomedical-engineering/3-d-mri-and-dt-mri-content-adaptive-finite-element-head-model-generation-forbioelectomagnetic-imagi

\section{INTECH}

open science | open minds

InTech Europe

University Campus STeP Ri

\section{InTech China}

Unit 405, Office Block, Hotel Equatorial Shanghai 
Slavka Krautzeka 83/A

51000 Rijeka, Croatia

Phone: +385 (51) 770447

Fax: +385 (51) 686166

www.intechopen.com
No.65, Yan An Road (West), Shanghai, 200040, China 中国上海市延安西路65号上海国际贵都大饭店办公楼405单元 Phone: +86-21-62489820

Fax: +86-21-62489821 
(C) 2009 The Author(s). Licensee IntechOpen. This chapter is distributed under the terms of the Creative Commons Attribution-NonCommercial-ShareAlike-3.0 License, which permits use, distribution and reproduction for non-commercial purposes, provided the original is properly cited and derivative works building on this content are distributed under the same license. 